\title{
«Der VSAO Schweiz verzichtet überraschend auf die Ergreifung des Referendums gegen die Verlängerung des Zulassungsstopps»
}

Diese Nachricht führte bei zahlreichen Kolleginnen und Kollegen zu entrüsteten Reaktionen begreiflicherweise.

Die Delegierten aller Sektionen kamen nach intensiver Auseinandersetzung mit der Frage zum Schluss, dass auf das Referendum gegen die Verlängerung des befristeten Zulassungsstopps $\mathrm{zu}$ verzichten sei und alle Energie auf den unmittelbar bevorstehenden Kampf gegen die Aufhebung des Kontrahierungszwangs (sogenannte Vertragsfreiheit) und einen gleichzeitig damit verbundenen zeitlich unbegrenzten Zulassungsstopp gerichtet werden soll. Dies bedeutet keineswegs, dass der VSAO Schweiz die Verlängerung des Zulassungsstopps billigt: Er ist und bleibt ein Armutszeugnis der schweizerischen Gesundheitspolitik!

Welche Strategie verfolgt denn nun der VSAO Schweiz für die Zukunft?

- Der VSAO Schweiz wird der kommenden Ärztekammer beantragen, dass ein erneuter Referendumsentscheid gegen die Vorlage Vertragsfreiheit und den damit einhergehenden Zulassungsstopp auf unbeschränkte Zeit (sic!) gefasst wird.

- Der VSAO Schweiz arbeitet aktiv an einer Vernetzung aller Partner für dieses Referendum und den folgenden Abstimmungskampf. Die Abstützung eines Referendums durch ein überparteiliches Komitee, in welchem neben der FMH und dem VSAO Schweiz eine breite
Koalition Einsitz nimmt, potenziert die Signalwirkung zugunsten eines paritätischen, qualitativ hochstehenden Gesundheitssystems!

- Wir klären unermüdlich Politiker, Versicherer und Gesundheitsökonomen darüber auf, dass es eine Fiktion ist, die Gesundheitskosten über die Anzahl Ärztinnen und Ärzte steuern zu wollen. Dies ist und bleibt eine Illusion - der Zulassungsstopp hat es bewiesen und wird es weitere drei Jahre zeigen!

- Gleichzeitig ist es Aufgabe der gesamten Schweizer Ärzteschaft, Alternativen zur Vertragsfreiheit, welche die Effizienz und Effektivität der Gesundheitsversorgung bei gleichbleibend hoher Qualität steigern, mit zu erarbeiten: Der VSAO Schweiz hat hier mit seinem «Positivmodell» (verschärfte positive Zulassungskriterien für Leistungserbringer, Analyse und Optimierung von Behandlungsprozessen, Pauschalierung von definierten Behandlungsprozessen) bereits wesentliche Vorarbeit geleistet.

Der Verzicht auf das Referendum ist ein positives Bekenntnis des VSAO Schweiz zu einem zukunftsgerichteten, konstruktiven Engagement gemeinsam mit der FMH - in der Gesundheitspolitik. Nicht mehr und nicht weniger!

Peter Studer, Präsident VSAO Schweiz 


\section{«L'ASMAC Suisse renonce à saisir le référendum contre la prolongation de la clause du besoin, à la grande surprise de tous»}

Cette nouvelle a soulevé une vague d'indignation chez de nombreux confrères, à juste titre, d'ailleurs.

Après d'âpres discussions, les délégués de toutes les sections ont décidé de renoncer au référendum visant à supprimer la prolongation provisoire du gel des admissions et à concentrer toutes les énergies sur la campagne imminente contre la levée de l'obligation de contracter (ou liberté de contracter) et contre le blocage qui en découle, d'une durée illimitée, de l'accès à la pratique privée. Il ne faut surtout pas en déduire que l'ASMAC Suisse approuve la prolongation du gel des admissions, qui est et restera un témoin des faiblesses de la politique suisse de la santé!

Quelle stratégie l'ASMAC Suisse va-t-elle maintenant adopter?

- L'ASMAC Suisse proposera à la prochaine Chambre médicale de saisir le référendum contre le projet relatif à la liberté de contracter et au blocage de l'accès à la pratique privée, d'une durée illimitée (sic!).

- L'ASMAC Suisse s'emploie activement à mettre en réseau les partenaires concernés par ce référendum et la campagne de vote s'y rapportant. Un comité hors partis et comprenant une large coalition, outre la FMH et l'ASMAC Suisse, s'en chargera et son importance renforcera le signal donné en faveur d'un système de santé paritaire et de qualité élevée!

- Nous affirmons sans cesse aux politiques, assureurs et économistes de la santé que vouloir maîtriser les coûts de la santé en agissant sur le nombre de médecins n'est que pure illusion et que le blocage à la pratique privée continuera de le prouver ces trois prochaines années!

- Simultanément, il incombe au corps médical suisse d'élaborer des solutions de rechange à la liberté de contracter, susceptibles d'augmenter l'efficacité de l'assistance sanitaire sans toucher à sa qualité. Avec son «modèle positif» (critères d'admission positivement renforcés pour fournisseurs de prestations, analyse et optimisation des processus thérapeutiques, forfaits pour certains traitements déterminés), l'ASMAC Suisse a déjà accompli un travail préparatoire important.

En renonçant au référendum, l'ASMAC Suisse adopte une attitude positive, indiquant qu'elle s'engage dans la politique de la santé, conjointement avec la FMH, pour y œuvrer d'une manière constructive et axée sur l'avenir. Ni plus ni moins!

Peter Studer, président de l'ASMAC Suisse 